\title{
Optical Properties of Tetrahedral Amorphous Carbon Films and Their Potential for Lab-on-a-Chip
}

\author{
Katja Guenther ${ }^{1 *}$, Frank Sonntag2 ${ }^{2}$, Teja Roch ${ }^{2,3}$, Andrés Fabián Lasagni ${ }^{2,3}$ \\ ${ }^{1}$ University of Applied Sciences Mittweida, Mittweida, Germany \\ ${ }^{2}$ Fraunhofer Institute for Material and Beam Technology IWS, Dresden, Germany \\ ${ }^{3} \mathrm{TU}$ Dresden, Institute of Manufacturing Technology, Dresden, Germany \\ Email: "guenther@creavac.de, Frank.Sonntag@iws.fraunhofer.de, Teja.Roch@iws.fraunhofer.de, \\ Andres-Fabian.Lasagni@iws.fraunhofer.de
}

Received 1 April 2015; accepted 24 May 2015; published 27 May 2015

Copyright (C) 2015 by authors and Scientific Research Publishing Inc.

This work is licensed under the Creative Commons Attribution International License (CC BY). http://creativecommons.org/licenses/by/4.0/

(c) (i) Open Access

\section{Abstract}

In this study, tetrahedral amorphous carbon (ta-C) films with thicknesses between several $100 \mathrm{~nm}$ and several micrometers have been deposited onto polished tungsten carbide and steel substrates by pulsed laser deposition (PLD) using an excimer laser ( $248 \mathrm{~nm}$ wavelength). We investigate the optical properties (e.g. the refractive index $(n)$ and extinction coefficient $(k)$ in the visible and near-infrared wavelength range) of these layers in dependence of the used laser ablation fluence on the target. It is shown that $n$ of $\sim 2000 \mathrm{~nm}$ thick ta-C films can be tuned, depending on the $\mathrm{sp}^{3}$-content, between $n=2.5$ and 2.8 at a wavelength of $632 \mathrm{~nm}$. Besides of this $k$ reduces with the $\mathrm{sp}^{3}$-content and is as low as 0.03 at $\mathbf{s p}^{3}$-contents of more than $\mathbf{7 5 \%}$. We proof that this gives the opportunity to prepare coating with tailored optical properties. Furthermore, it is shown that the ta-C films have low background fluorescence in the wavelengths range of $380-750 \mathrm{~nm}$, which make this thin films attractive for certain optical, medical and biotechnological applications. We present for the first time that one possible application is the use in Lab-on-a-Chip-systems (LOC). Within these systems, the ultrasensitive detection of fluorescence markers and dyes is a challenge. In order to increase the signal-to-noise-ratio, a setup was developed, that used the specific optical properties of ta-C films produced by PLD. We used the ta- $\mathrm{C}$ film as an integrated reflector that combined low background fluorescence, a low reflectivity at the excitation wavelength and the high reflectivity at the emission wavelength. We prove that this setup improves the detection of fluorescence photons.

"Corresponding author.

How to cite this paper: Guenther, K., Sonntag, F., Roch, T. and Lasagni, A.F. (2015) Optical Properties of Tetrahedral Amorphous Carbon Films and Their Potential for Lab-on-a-Chip. Materials Sciences and Applications, 6, 445-455. 
Keywords

Tetrahedral Amorphous Carbon, Pulsed Laser Deposition, Thin Films, Diamond-Like Carbon, Lab-on-a-Chip

\section{Introduction}

Diamond-like carbon films (DLC), especially the group of super-hard, hydrogen-free amorphous carbon films (ta-C) with a high content of tetrahedral bonding, are used in a lot of industrial applications, actually. Most applications like wear protection, anti-corrosion and friction reduction of tools and machine parts benefit from the mechanical properties of the ta-C films, like a high hardness in the range of 40 - $90 \mathrm{GPa}$ [1] and a high Young's modulus in the range of $200-820 \mathrm{GPa}$ [1] as well as low coefficients of friction in the range of 0.1 - 0.2 [2]-[5].

Applications in biology or optics benefit from the high biocompatibility (e.g. biosensor applications [6]) and characteristic reflectivity of the ta-C films (e.g. anti-reflection coating [7]).

For ta-C thin film deposition it is well known, that the energy of the film forming species influences the content of tetrahedral bindings ( $\mathrm{sp}^{3}$-fraction). The physical properties of ta-C films depend mainly on the $\mathrm{sp}^{3}$-fraction. An increase of the $\mathrm{sp}^{3}$-content causes an increase of hardness, elasticity modulus and simultaneously an increase in transparency in the visible and near infrared range (e.g. [5] [7]-[9]).

There are already different publications on the optical properties of ta-C. Exemplary Tay et al. [10] have measured the optical properties of ta-C films deposited onto silicon substrates using ion energies between 35 and $195 \mathrm{eV}$. These ta-C films exhibit refractive indices $(n)$ between 2.4 and 2.9 and an extinction coefficient $(k)$ between nearly 0 and 0.25 in a wavelength range of about 200 to $900 \mathrm{~nm}$. The optical energy-gap varied between 2.1 and $2.6 \mathrm{eV}[10]$.

Additionally Mathioudakis et al. [8] presented calculations onto the relation between disorder, $\mathrm{sp}^{3}$-content and optical properties inamorphous carbon films. They calculated avariation of the optical band gap between nearly 0 and $5 \mathrm{eV}$ in dependence on the $\mathrm{sp}^{3}$-fraction. According to Patsalas [7] DLC-films with increased $\mathrm{sp}^{3}$-fraction have also an increased density $\left(1.8\right.$ to nearly $\left.3.3 \mathrm{~g} / \mathrm{cm}^{3}\right)$.

Furthermore Panwar [11]-[13] analyzed the influences of substrate bias, hydrogen and nitrogen content onto the reflectance, the photoluminescence and the $\mathrm{sp}^{3} / \mathrm{sp}^{2}$-ratio. The seta-C-, ta-C:N- and ta-C:H-films were deposited by an S-bend filtered cathodic vacuum arc process. Ta-C films produced with this method show no interference effects in the wavelength range $300-600 \mathrm{~nm}$. The reflectance values vary between $10 \%$ - 50\% [11].

It is well known, that ta-C films can be produced using different PVD-methods like $\mathrm{S}$ bend filtered cathodic arc [11]-[13], pulsed laser vacuum arc [4] [9] or pulsed laser deposition (PLD) [14]. PLD has the advantage of low droplet formation in comparison to e.g. an arc process as well as a good controllability of the energy of the film forming species.

One possible optical application for ta-C films is the integration onto Lab-on-a-Chip systems (LOC). LOC systems offer a high degree of integration, miniaturization, automation and the realization of complex processes. The potential of LOC systems is growing in many areas of biotechnology and medical engineering [15] [16]. Basically all LOC systems consist of an immobilized biological component (e.g. DNA, antibodies, enzymes, cells) at the edge of a transducing device like electrodes or optical components like transparent windows or wave guides. The biggest advantage of optical measurements is the ability to detect low quantities of target molecules without direct probe contact. Among those photometric detection approaches fluorescence measurement is the most common method. One reason is the wide range of fluorescent dyes that allow the detection of target molecules at almost any wavelength from UV up to near infrared. Moreover only small sample volumes are needed. Nevertheless in most cases fluorescence dyes are designed for special excitation wavelengths, which are available in almost any fluorescence microscope, flow cytometer or plate reader. Using these benefits of fluorescence many LOC applications were built up in the past [17]. Thereby, the ultra-sensitive detection of fluorescence markers and of dyes is one challenge in LOC applications. Nevertheless still an open issue is to increase photoluminescence efficiency and a reduction of the signal to noise ratio.

In this study we want to present an approach where ta-C is discussed as a dispersive reflector in LOC. To gain an extended understanding of the optical properties of the ta-C films deposited by PLD, we investigated the in- 
fluence of the thin film substrates. Further investigations are made about the influence of the laser ablation fluence during film deposition and the deposited ta-C-film thickness onto the optical properties of ta-C.

Based on these results a setup will be shown, which uses the special optical properties of an integrated ta-C thin film reflector. This setup increases the signal-to-noise-ratio in a LOC system. Such an integrated ta-C film based reflector combines low background fluorescence, low reflectivity at the excitation wavelength and high reflectivity at the emission wavelength. Therefore we investigate the rising of the sensitivity of fluorescence measurements by using biocompatible ta-C films onto polished steel or tungsten carbide substrates as reflector.

\section{Material and Methods}

\subsection{PLD Deposition of ta-C Films}

The analyzed ta-C layers were mainly prepared by using the PLD method. Some films were deposited using a combination of PLD and pulsed laser annealing (also known as laser stress relaxation or LSR), the latter resulting in stress relaxation. The experimental set-up is shown in Figure 1.

The ta-C films have been prepared onto steel and tungsten carbide substrates possessing an average roughness $(R a)$ below $10 \mathrm{~nm}$ and maximum dimensions of $40 \mathrm{~mm}$ diameter and $5 \mathrm{~mm}$ thickness. In comparison to steel, tungsten carbide is robust against disinfection and autoclaving.

In order to ensure good adhesion of the ta-C layers, the substrates have been cleaned in an ethanol ultrasonic bath up to 5 min before placing them into a high vacuum chamber. In a second step the substrates have been bombarded with an argon ion beam produced in a Kaufmann ion source immediately prior to the deposition in the vacuum chamber ( $1 \mathrm{~min}, 10^{-2} \mathrm{~Pa}$ working pressure, $700 \mathrm{eV}$ ion energy and nearly $150 \mu \mathrm{A} / \mathrm{cm}^{2}$ current density onto the substrate). Additionally an $80 \mathrm{~nm}$ thick tungsten carbide adhesion layer was prepared by PLD. Therefore a Krypton fluoride excimer $(\mathrm{KrF})$ laser (248 nm wavelength; $25 \mathrm{~ns}$ pulse duration, max. $1 \mathrm{~J}$ pulse energy, up to $50 \mathrm{~Hz}$ pulse repetition rate) operating with an unstable resonator was used for the ablation of a tungsten carbide target by utilizing ablation fluences of $12 \mathrm{~J} / \mathrm{cm}^{2}$ onto the target surface.

The same laser was used for the ablation of a graphite target, i.e. for the deposition of the ta-C films. The ta-C films have been prepared using various laser fluences in the range of $2-22 \mathrm{~J} / \mathrm{cm}^{2}$ for the graphite target ablation. The stress relaxation was made by using LSR every $100 \mathrm{~nm}$ Sublayer. The used LSR-laser was a second $\mathrm{KrF}$ laser (as described before) but operating with a stable resonator. The annealing was performed with a laser pulse fluences of about $90 \mathrm{~mJ} / \mathrm{cm}^{2}$ and a maximum number of laser pulses per unit area of about 200. Using the LSR method nearly stress-free, super-hard ta-C films with thicknesses of several micrometers can be prepared. The residual gas pressure during deposition was $1 \times 10^{-4} \mathrm{~Pa}$. The maximum temperature at the substrate was below $100^{\circ} \mathrm{C}$ and no substrate bias was added. The entire process and further details can be found in Ref. [14].

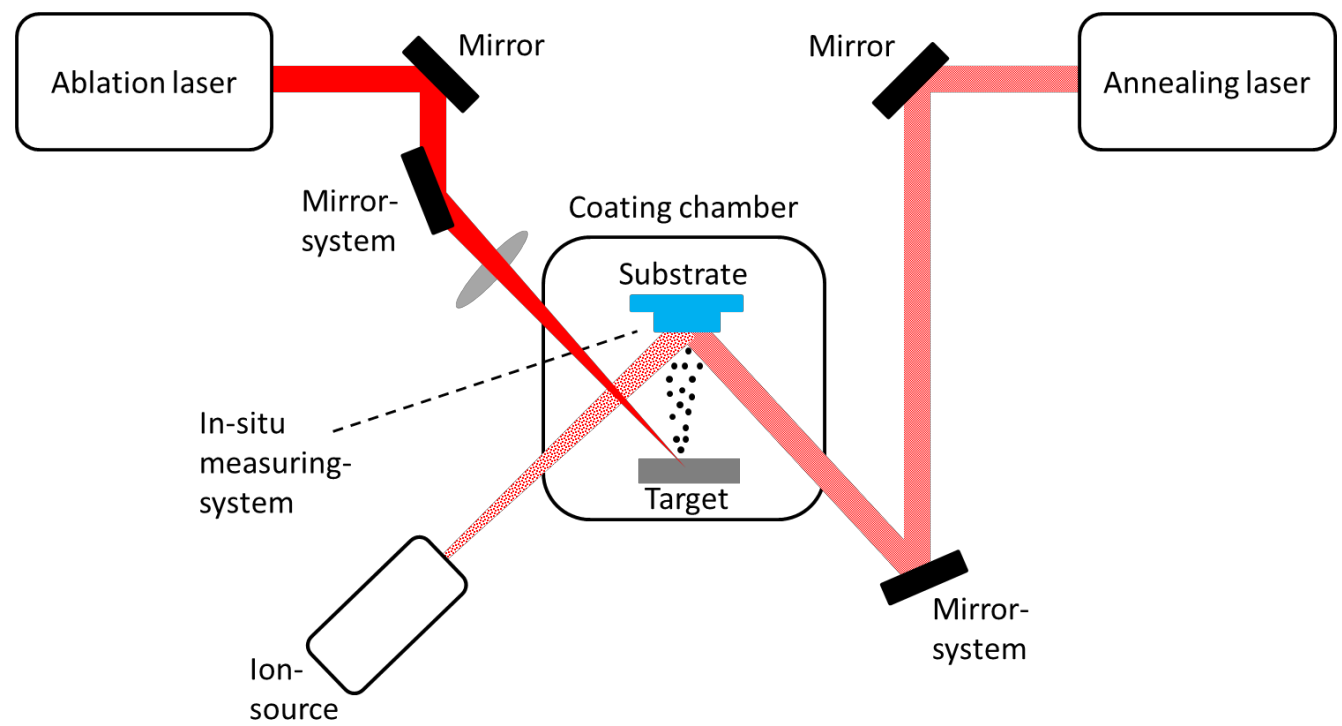

Figure 1. Experimental set-up used for the preparation of the ta-C films. 
Using PLD without LSR the stress of a $2000 \mathrm{~nm}$ ta-C film up to $10 \mathrm{GPa}$. Using the combination of PLD and LSR it is possible to reduce the internal stress to nearly $0 \mathrm{GPa}$ of such a ta-C film. In this work ta-C films which were produced with PLD show internal stresses between 8 - $10 \mathrm{GPa}$ and those which were produced with PLD and LSR show internal stress between 0 - 2 GPa.

\subsection{Thin Film Characterization Methods}

The surface roughness and the film thickness were measured using a profilometer DEKTAK 3030 from Veeco instruments. In that case the film thickness was measured at a film-edge.

The refractive index $(n)$, the absorption index $(k)$ and the reflectivity $(R)$ of the coated substrates were investigated using the spectrometer F20 from Filmmetrics and the software "FILMeasure". The reflectance measurements were carried out by irradiating the substrates with wavelengths in the range between 380 - $1050 \mathrm{~nm}$ vertical to the substrate surface (angle of incidence $0^{\circ}$ ).

The internal stress of the ta-C films was measured once in situ using a position sensitive detector and twice ex situ using a surface profilometer. Both methods measure the deflection of a thin silicon wafer, which was inserted into the vacuum chamber next to the substrate. With the Stoney equation the internal stress of the ta-C films deposited onto the silicon substrate was calculated.

The $\mathrm{sp}^{3} / \mathrm{sp}^{2}$-content was calculated using databased on nano-indentation-hardness-measurements (10 mN loads) and LaWavemeasurement.

\subsection{Lab-on-a-Chip Device}

The fluorescence measurements were performed by a microscope (Zeiss LSM5 Pascal, light source: Zeiss HBO100, filter set: 9 and 15, detector: CCD camera) and a compact system developed by Fraunhofer Institute of Applied Optics and Precision Engineering IOF in cooperation with Fraunhofer Institute for Material and Beam Technology IWS [17]. For the excitation a high power LED with filter $479 \pm 20 \mathrm{~nm}, 531 \pm 20 \mathrm{~nm}$ or $585 \pm 20$ $\mathrm{nm}$ where integrated into a microscope similar optical system and used as the light source. The excitation wavelength is separated from the fluorescence signal by a filter $524 \pm 20 \mathrm{~nm}, 641 \pm 38 \mathrm{~nm}$ or $628 \pm 20 \mathrm{~nm}$. For the detection of the fluorescence signal a photomultiplier (Hamamatsu H10723-20) with adjustable amplification was used [17].

The fluorescenceirradiation is emitted nearly isotropic in all spatial directions. A sensitive detection system was used to record data of a wide dihedral angle. The optical system works with a high numerical aperture (NA) and thus an aspherical front lens (NA 0.5) is used. The optical imaging shows illuminated channels (analyzed volume) with a maximum width of $0.5 \mathrm{~mm}$ (Figure 2). To increase the sensitivity of the fluorescencesignal, at the up side of the analyzed volume should be a cover (reflector), which reflects the emitted fluorescenceirradiation in direction of the detector (Figure 2).

The reflector should possess the following properties:

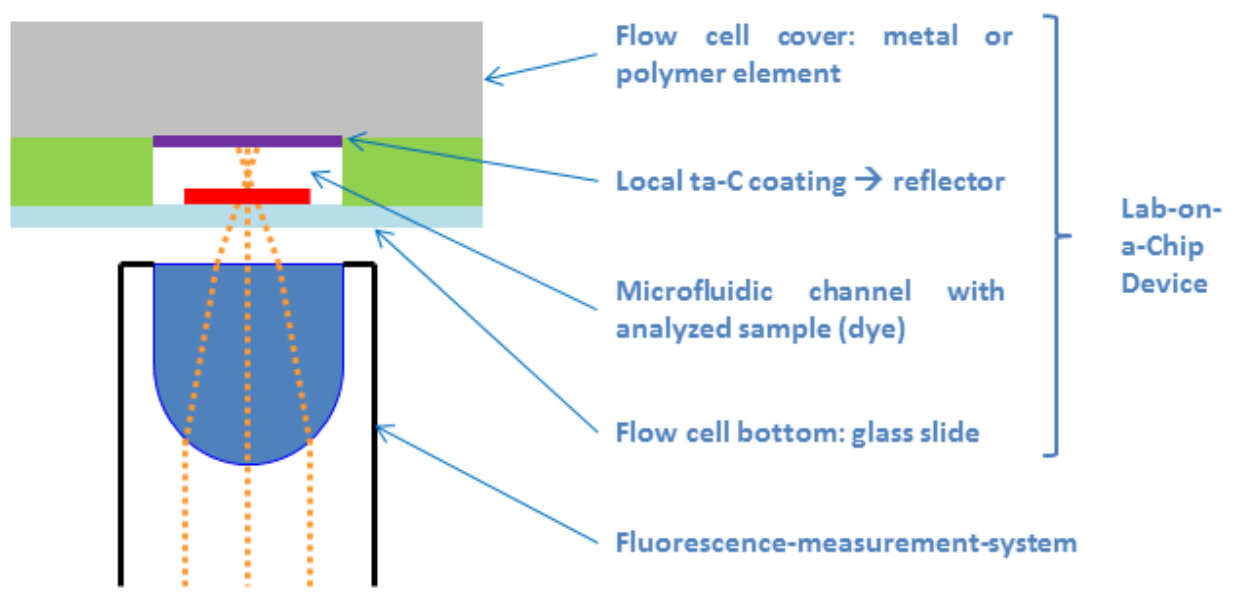

Figure 2. Schematic sketch of the real fluorescence-measurement-system-setup. 
- High biocompatibility (no toxicity for human cells and no immune reaction);

- Low fluorescence at the used excitation wavelength;

- Low reflectivity of the used excitation wavelength;

- High reflectivity of the generated emission wavelength.

\section{Theory}

The following optical analyses are based on the physical effect of thin film interference. Considering a homogeneous thin film the transmission $T$ depends on the film thickness $(d)$ and the absorption coefficient $(\alpha)$. This dependence is described by the Lambert-Beer-Law $(T=\exp (-\alpha \cdot d))$. However, if $\alpha \cdot d>1$ multiple reflections of the light within the thin film might occur. In this case the light reflected at the front surface of the ta-C interacts with light within the thin film. Thus the light can interfere either constructively, increasing the reflection or destructively, increasing the film absorption. This thin film interference depends on the phase difference of both light waves and is determined by the wavelength $\lambda$ and the optical path length in the thin film.

It is well known, that the optical path length in the thin film depends on the layer thickness $d$, the refractive index $n$ and the angle of incident. The position of constructive and destructive interference varies according to [18]:

$$
\begin{aligned}
& \text { Constructive interference: } 2 d n_{1}=m \cdot \lambda \quad m=1,2,3, \cdots \\
& \text { Destructive interference: } 2 d n_{1}=\left(m+\frac{1}{2}\right) \lambda \quad m=0,1,2, \cdots
\end{aligned}
$$

Formula (1) and Formula (2) are valid on the following terms:

- Measurements are undertaken in air. $n$ of air is presumed with the value 1 ;

- $\quad n$ of the substrate has higher values than the film;

- The surface is irradiated perpendicularly;

- The layer is smooth and has a homogeneous thickness.

\section{Results and Discussion}

\subsection{Optical Properties of the Substrates}

Because of the low $R a<10 \mathrm{~nm}$, the analyzed tungsten carbide substrates possess a nearly constant $R$ of about nearly 44\% in the specified measurement range (380 - $1050 \mathrm{~nm})$. The $R$ of the steel substrate is comparable higher $(R=64 \%)$. Hence these substrates are useful for optical investigations based on interference effects of ta-C films.

\subsection{Influence of the Used Ablation Fluence onto the Optical Properties of ta-C Films}

Figure 3 shows the measured $R$ of $\sim 2000 \mathrm{~nm}$ thick ta-C films deposited onto tungsten carbide substrates using PLD with different ablation fluences $(F)$ and no LSR.

Using PLD ablation fluences between $5.3-21.7 \mathrm{~J} / \mathrm{cm}^{2}$ to deposit ta-C films all curve progressions show typical interference-maxima and -minima for partly transparent films in the measurement range (Figure 3). The maxima and minima appear at different wavelengths, because of differences in $n$ and the film-thicknesses. Further experimental investigations will be shown in Section 4.3. Using PLD ablation fluences below $5.3 \mathrm{~J} / \mathrm{cm}^{2}$ maxima and minima in all curve progressions are nearly vanished (Figure 3).

Uncoated tungsten carbide substrates have $\sim 4 \%$ reflectivity in the analyzed wavelength range. In comparison tungsten carbide substrates coated with $\sim 2000 \mathrm{~nm}$ thick ta-C deposited by PLD using ablation fluences above $5.3 \mathrm{~J} / \mathrm{cm}^{2}$ show $R$ values between $2 \%$ and $44 \%$ depending on the wavelength. Using ta-C coatings prepared with lower laser fluencies $\left(<5.3 \mathrm{~J} / \mathrm{cm}^{2}\right.$ the $R$ is reduced to $20 \%$ - 26\% over the whole analyzed wavelength range. A similar behavior can be observed for steel substrates.

Investigating the optical properties of $2000 \mathrm{~nm}$ thick ta- $\mathrm{C}$ on tungsten carbide we found that an increase of the ablation fluence causes a decrease of $n$ from about 2.8 to 2.5 and a decrease of $k$ from about 0.7 to 0.03 , respectively (see Figure 4(a) and Figure 4(b)). Similar results were obtained using steel substrates.

This behavior results from the $\mathrm{sp}^{3}$-fractionwhich corresponds to the hardness of the ta-C films. Ta-C films 


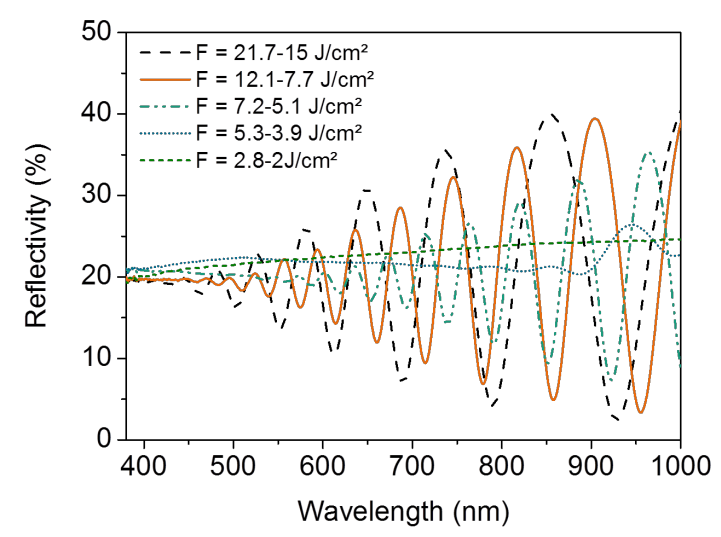

Figure 3. $R$ of ta-C-films deposited onto tungsten carbide substrates using different PLD ablation fluencesw.
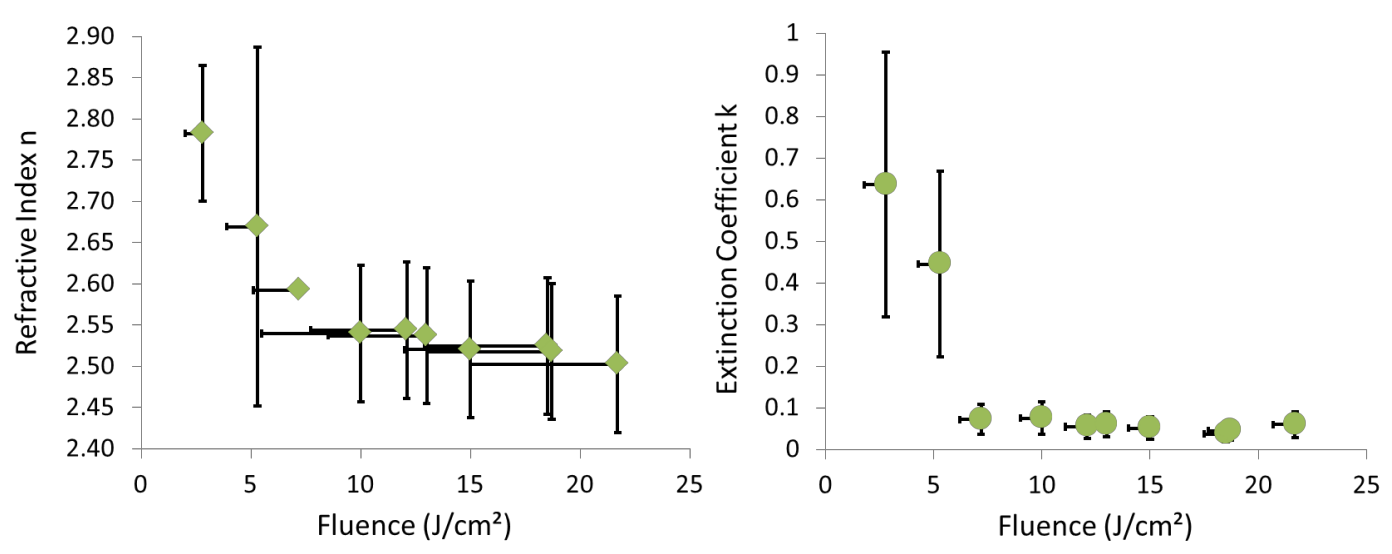

Figure 4. (a) Refractive index $n$ and (b) exctiction coefficient $k$ (at $632 \mathrm{~nm}$ ) in dependence of the used PLD ablation fluence $F$.

generated with PLD ablation fluences above $5.3 \mathrm{~J} / \mathrm{cm}^{2}$ possess a predominant $\mathrm{sp}^{3}$-bonded atomic network and thus resulting innanoindentation hardness's between $30 \mathrm{GPa}$ and $63 \mathrm{GPa}$. Whereas ta-C films deposited using PLD ablation fluences below $5.3 \mathrm{~J} / \mathrm{cm}^{2}$ show graphitic film growth with a predominant $\mathrm{sp}^{2}$-fraction. The nanoindentation hardness of these films is below $25 \mathrm{GPa}$.

The transition point between dominantly $\mathrm{sp}^{2}$-bonded or $\mathrm{sp}^{3}$-bonded amorphous film growths was determined at PLD ablation fluence about $5.3 \mathrm{~J} / \mathrm{cm}^{2}$. At this point the measurement of $n$ and $k$ has large measuring errors. Hence increase of the $\mathrm{sp}^{3}$-fraction results in a preference of the optical transparence of the ta-C films i.e. the reduction of $k$. The higher the $\mathrm{sp}^{3}$-fraction the more the $n$ should approximate to values of pure diamond ( $n$ nearly 2.42).

Because of the amorphous structure and the mixture of $\mathrm{sp}^{2}$ - and $\mathrm{sp}^{3}$-bonding, it is not possible to reach transparencies of pure diamond ( $\mathrm{sp}^{3}$-fraction $100 \%$ ) for $2000 \mathrm{~nm}$ thick ta-C films. Ta-C films with a low $\mathrm{sp}^{3}$-fraction are characterized by an increased absorption, become opaque no thin film interference can be observed. Further investigations will be shown in the Dissertation of K. Günther [19]. These results are also consistent with data from several authors in the literature which have published major articles about the relation between $\mathrm{sp}^{3}$-content and the optical properties of ta-C [7] [8] [10] [13].

\subsection{Optical Properties in Dependence on the ta-C Film Thickness}

Figure 5 shows $R$ of ta-C films with a variable film thickness deposited onto tungsten carbide using PLD ablation fluences about $12 \mathrm{~J} / \mathrm{cm}^{2}$ and optimizes LSR (internal stress below $1 \mathrm{GPa}$ ). Expectedly the increase of the film thickness results in a minimization of the distance between two consecutive amplitudes of the interference maxima/minima. The relation is based on formula 1 and 2 (Section 3). The higher the film thickness, the more 


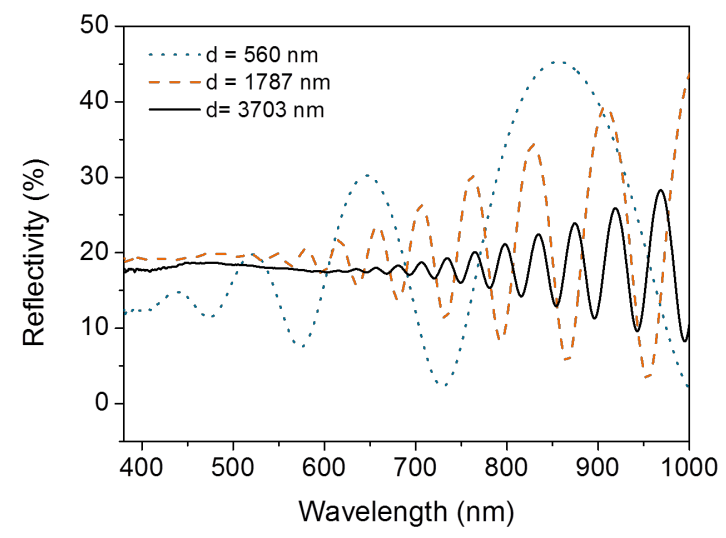

Figure 5. $R$ of ta-C films with a variable film thickness deposited onto tungsten carbide using PLD ablation fluence of $12 \mathrm{~J} / \mathrm{cm}^{2}$.

the position of the maxima will slide to higher wavelength. This effect can be used to produce wavelength selective constructive or destructive interferences in dependence on the ta-C film thickness (Section 4.5.3) [19].

Further investigations show a decrease of $\mathrm{n}$ from 2.55 to 2.47 with increasing ta-C film thickness from nearly 2000 to around $3800 \mathrm{~nm}$. However $k$ shows low values between 0.07 and 0.1 for the inspected ta-C film thicknesses. Furthermore the amplitudes of the interference maxima and minima are reduced with increasing ta-C film thickness. The same results were obtained using steel substrates.

One possible reason for decreasing $n$ at ta-C films with a film thickness above $3500 \mathrm{~nm}$ could be the rising influence of defects with increase of the film thickness. Droplets/particulates will outgrow of the ta-C film surface. This results in a higher deflection of the incident measurement irradiation. Furthermore defects can produce a deflection at their edges. The deflected irradiation fraction cannot be recorded from the detector and is therefore lost. So the calculated $n$ could have high faults.

\subsection{The Influence LSR onto the Optical Properties of ta-C Films}

Most applications for ta-C films are in the film thickness range from some $100 \mathrm{~nm}$ up to several micrometers. Especially in the case of using steel substrates it is important to deposit ta-C films with low internal stress to avoid the film from peeling off the substrate. Using PLD in combination with LSR, it is possible to reduce the internal stress of the ta-C films close to 0 GPa without phase transformation.

Figure 6 shows exemplary equal curve progressions for $R$ of two nearly $1200 \mathrm{~nm}$ thick ta-C films onto steel substrates. One film was deposited using only the PLD and the other with the same PLD parameters but in combination with LSR. Thus one sample has maximum internal stress between 8 - $10 \mathrm{GPa}$ and the other (PLD/LSR deposited) depicts internal stresses close to $0 \mathrm{GPa}$.

The refractive index of both samples is comparable $\left(n_{\mathrm{PLD}}=2.602, n_{\mathrm{PLD} / \mathrm{LSR}}=2.622\right)$ and in the range of measuring error $( \pm 0.02)$.

On closer examination Figure 6 show a shift between the curve progressions of these two ta-C films. This effect is the result of a minimal difference in the film thickness, explained in section 3 and 4.3. The not stress relaxed film has a thickness of about $1175 \mathrm{~nm}$ and the stress relaxed one has a film thickness of about $1198 \mathrm{~nm}$.

The same results were obtained using tungsten carbide substrates.

Thus the application of the LSR does not influence the optical properties of ta-C films with a high $\mathrm{sp}^{3}$-fraction. Therefore the LSR process is suitable to deposit ta-C films with high optical transparency and mechanical properties like a high hardness.

\subsection{Potential of ta-C Films in the Application LOC}

4.5.1. Background Fluorescence Measurements onto Different Reflectors without Using Markers First tests regarding fluorescence signals of flat uncoated and coated reflectors were taken out using the set-up shown in Figure 2 using an uncoated glass slide, without any dyes. Therefore the highest possible sensitivity of 


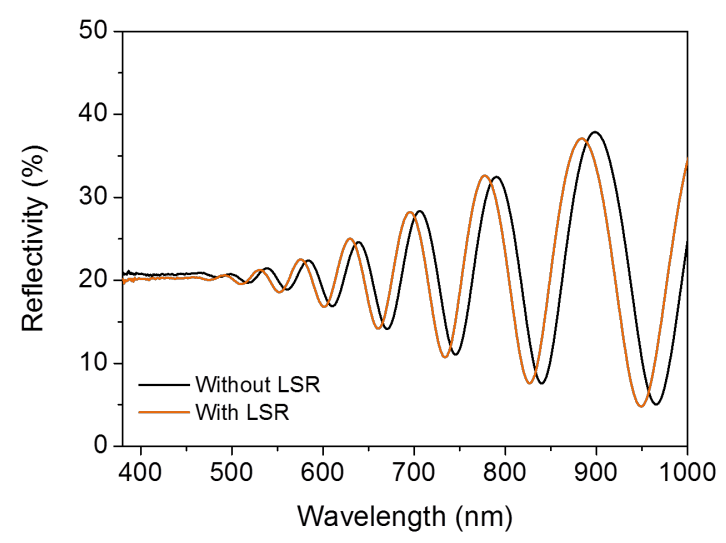

Figure 6. Comparison between curve progressions of $R$ of nearly $1200 \mathrm{~nm}$ thick ta-C films deposited using a combination of PLD/LSR.

the photomultiplier and conditions as shown in Table 1 were utilized. The mean value of the fluorescence signal was estimated from 100.000 measured values.

In all cases the analyzed reflectors show minimal fluorescence values at the different excitation wavelengths, which are equal to those of gold. The fluorescence signal for ta-C films shows values between 0.09 and $0.10 \mathrm{~V}$ (excitation wavelength $479 \pm 20 \mathrm{~nm}$, detection wavelength $524 \pm 20 \mathrm{~nm}$ ), independent of the used LSR process or used ablation fluence onto the target during the film deposition.

This means, that the ta- $C$ coating insulates the fluorescence of basic material from the detection. Hence the influence of the excitation wavelength onto the measurement signal can be eliminated using ta-C films.

\subsubsection{Fluorescence Measurements Using Markers and Different Reflectors}

In a further step fluorescence measurements of a fluorescence marker were taken out by replacing the uncoated glass slide with a fluorescence-marker-coated glass slide (Figure 2).

The signal of the fluorescence irradiation emitted by the marker was measured at a fixed position without and with a reflector in a distance of about $500 \mu \mathrm{m}$. For a better visualization the amplification of the measured fluorescence signal is described subsequent using an amplification-factor $(A)$ :

$$
A=\text { sensor signal with reflector/sensor signal without reflector }
$$

Using a reflector increases the sensor signal (using maximal sensitivity) compared to measurements which were taken out without any reflector for all analyzed reflectors and excitation/detection wavelengths. Figure 7 shows exemplary at an excitation wavelength of about $479 \pm 20 \mathrm{~nm}$ and detection wavelength $524 \pm 20 \mathrm{~nm}$ for the analyzed reflectors. In consequence it is on the one hand possible to increase the signal to noise ratio. On the other hand the coating gives the opportunity to suppress the excitations of underlying layers.

In comparison to the gold or the uncoated tungsten carbide reflector $A$ of the nearly $2000 \mathrm{~nm}$ thick ta-C films is lower. This effect results from $R$ of the used reflectors (Figure 8).

Gold has $R$ of about $80 \%$, tungsten carbide of $44 \%$ and $2000 \mathrm{~nm}$ ta-C films on tungsten carbide of nearly $20 \%$ at the detection wavelength of about $524 \pm 20 \mathrm{~nm}$. Due to that fact it is possible to optimize $R$ of the reflectors for defined wavelength. Using ta-C films with a high $\mathrm{sp}^{3}$-fraction the effect of generating interference maxima and minima at well-defined wavelength can be used for wavelength selective amplification of the measured fluorescence signal.

\subsubsection{Example for an Optimized Combination out of ta-C Film Thickness and Marker}

Based on Rat the analyzed wavelength range useful combinations of film-marker were determined. Figure 9 shows exemplary a combination of a1000 nm thick ta-C film on steel and the fluorescence marker Cy7 (BD Biosciences) or QDOT800 (Nanocrystal). The excitation wavelength for these markers is in the range of an interference minimum in the curve progression of $R$ of the $1000 \mathrm{~nm}$ thick ta-C film, whereas the detection wavelength of these markers is at an interference maximum (Figure 9). 
Table 1. Test conditions during the fluorescence measurements.

\begin{tabular}{cc}
\hline Parameter & Value \\
\hline Channel height & $500 \mu \mathrm{m}$ \\
Excitation wavelength & $567 \mathrm{~nm} \pm 20 \mathrm{~nm}$ \\
Metection or fluorescence wavelength & $530 \mathrm{~nm} \pm 20 \mathrm{~nm}$ \\
Marker & Mixture consisting of different laser dyes embedded into a \\
homogeneous polymer film at the glass slide
\end{tabular}

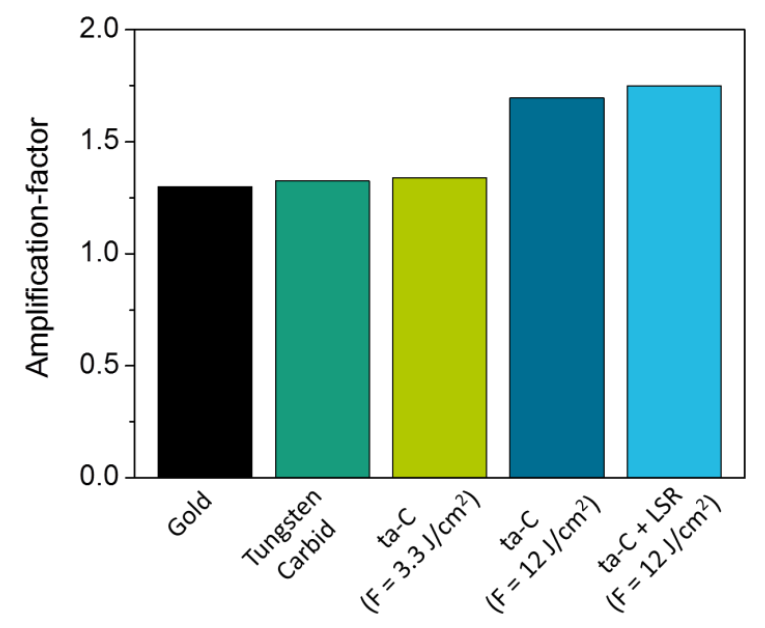

Figure 7. Amplification-factor $(A)$ of $~ 2000 \mathrm{~nm}$ thick ta-C films deposited onto tungsten carbide using different deposition parameters in comparison with gold- and tungsten carbide reflector. The excitation wavelength was $479( \pm 20) \mathrm{nm}$ and the detection wavelength $524( \pm 20) \mathrm{nm}$.

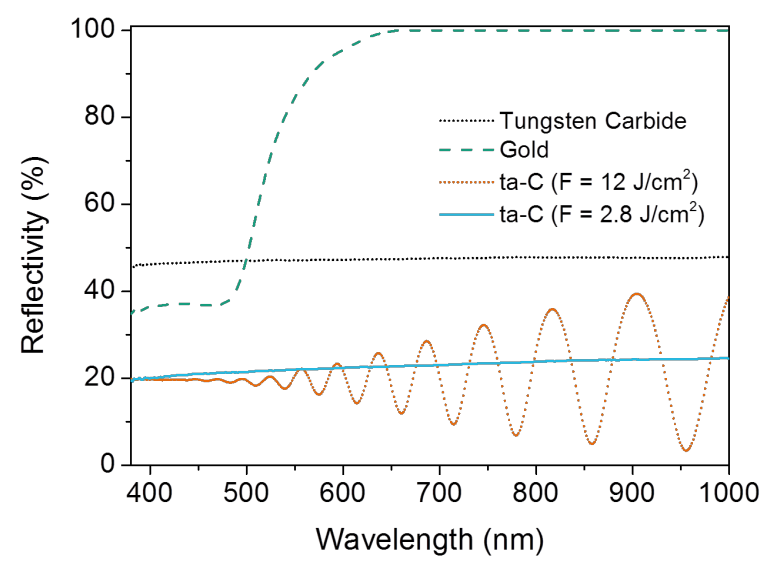

Figure 8. $R$ curve progression of different reflectors in the wavelength range $380 \mathrm{~nm}-1050 \mathrm{~nm}$.

\section{Conclusions}

In this study the optical properties of ta-C films deposited by PLD have been analyzed. It is shown, that $R$ of several $\mu \mathrm{m}$ thick ta-C films does not depend on the used tungsten carbide or steel substrate, if $R a$ of the substrates possesses values below $10 \mathrm{~nm}$.

Further investigations show the reduction of $n(2.8-2.5)$ and simultaneously of $k(0.7-0.03)$ caused by the 


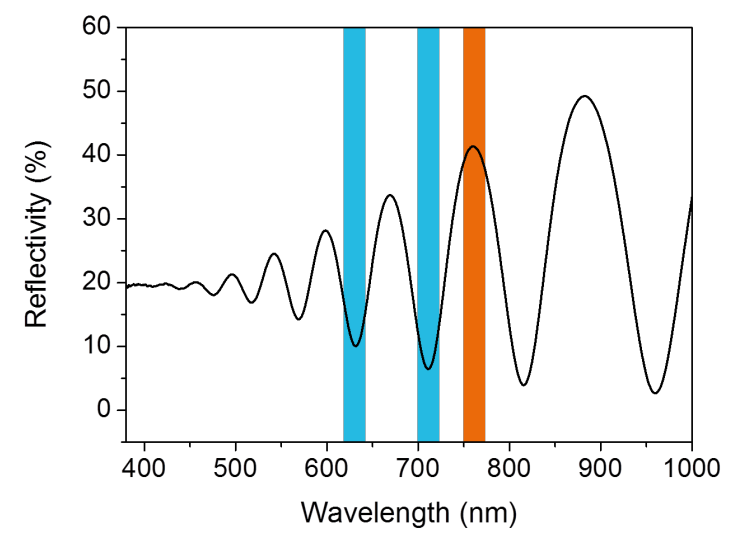

Figure 9. Exemplary $R$ for a nearly $1000 \mathrm{~nm}$ thick ta-C film deposited by PLD onto tungsten carbide substrate and marked wavelength ranges for excitation (grey) and fluorescence-emission (dark grey) for the fluorescence marker Cy7.

increase of the used ablation fluence during the PLD process. Consequently a rising $\mathrm{sp}^{3}$-fraction of the ta-C films with increasing the ablation fluence can be reasoned.

Ta-C films with a high $\mathrm{sp}^{3}$-fraction generate interference maxima and minima, if the ablation fluence during deposition is higher than $5.3 \mathrm{~J} / \mathrm{cm}^{2}$. The possibility to form interference maxima/minima gives the opportunity for an application as high refractive films for reflectivity minimization or reflectivity increase in the visible and near infrared wavelength range. Furthermore it is conceivable to use ta-C films with a high $\mathrm{sp}^{3}$-contant for decorative and at once wear resistant applications like jewelry or infrared optics.

Ta-C films with high $\mathrm{sp}^{2}$-content, in our case deposited by fluences below $5.3 \mathrm{~J} / \mathrm{cm}^{2}$, could be used as antireflection coatings in the wavelength range $380-1050 \mathrm{~nm}$. The transition point between graphitic and diamond film growth was determined at an ablation fluence of about $5.3 \mathrm{~J} / \mathrm{cm}^{2}$.

The mechanical and optical parameters, like $n$ and $k$ of ta-C films can be modified using different ablation fluences. Further investigations exhibit no influence of the used LSR process onto the optical properties of ta-C films.

The implementation of ta-C film based reflectors in a LOC system shows that it is possible to minimize background fluorescence from flow cell and cover. Furthermore it is possible to increase wavelength-selective the emitted fluorescence signal and decrease the excitation wavelength signal using ta-C coated channel walls. One great advantage in using ta-C films with high $\mathrm{sp}^{3}$-content in LOC is the minimization of the costs in comparison to using gold layers. To increase the measured signal of the fluorescence in LOC systems, further investigations concerning multilayers are promising.

\section{Acknowledgements}

This work has been technical and material supported by the University of Applied Sciences Mittweida and the Fraunhofer Institute for Material and Beam Technology IWS Dresden. The author gratefully acknowledges Steffen Weißmantel and Rene Eger.

\section{References}

[1] (2012) Kohlenstoffschichten-Grundlagen, Schichttypen und Eigenschaften: VDI2840-2012-06, in VDI-Handbuch Produktionstechnik und Fertigungsverfahren, Band 3, Betriebsmittel VDI-Handbuch Werkstofftechnik Verein Deutscher Ingenieure Beuth, Verlag GmbH, Berlin.

[2] Charitidis, C.A. (2010) Nanomechanical and Nanotribological Properties of Carbon-Based Thin Films: A Review. International Journal of Refractory Metals and Hard Materials, 28, 51-70. http://dx.doi.org/10.1016/j.ijrmhm.2009.08.003

[3] Kang, M.C., Tak, H.S., Jeong, Y.K., Lee, H.W. and Kim, J.S. (2010) Properties and Tool Performance of ta-C Films Deposited by Double-Bend Filtered Cathodic Vacuum Arc for Micro Drilling Applications. Diamond and Related Ma- 
terials, 19, 866-869. http://dx.doi.org/10.1016/j.diamond.2010.02.002

[4] Schulz, H., Scheibe, H.-J., Siemroth, P. and Schultrich, B. (2004) Pulsed Arc Deposition of Super-Hard Amorphous Carbon Films. Applied Physics, A78, 675-679. http://dx.doi.org/10.1007/s00339-003-2280-8

[5] Neuville, S. and Matthews, A. (2007) A Perspective on the Optimization of Hard Carbon and Related Coatings for Engineering Applications. Thin Solid Films, 515, 6619-6653. http://dx.doi.org/10.1016/j.tsf.2007.02.011

[6] Yu, H., Li, P. and Robertson, J. (2011) Fabrication and Bio-Functionalization of Tetrahedral Amorphous Carbon Thin Films for Bio Sensor Applications. Diamond and Related Materials, 20, 1020-1025. http://dx.doi.org/10.1016/j.diamond.2011.06.005

[7] Patsalas, P. (2011) Optical Properties of Amorphous Carbons and Their Applications and Perspectives in Photonics. Thin Solid Films, 519, 3990-3996. http://dx.doi.org/10.1016/j.tsf.2011.01.202

[8] Mathioudakis, C., Kopidakis, G., Patsalas, P. and Kelires, P.C. (2007) Disorder and Optical Properties of Amorphous Carbon. Diamond and Related Materials, 16, 1788-1792. http://dx.doi.org/10.1016/j.diamond.2007.08.009

[9] Schultrich, B. (2011) Modeling of ta-C Growth: Influence of the Technological Parameters. Diamond and Related Materials, 20, 785-792. http://dx.doi.org/10.1016/j.diamond.2011.03.035

[10] Tay, B.K., Shi, X., Cheah, L.K. and Flynn, D.I. (1997) Optical Properties of Tetrahedral Amorphous Carbon Films Determined by Spectroscopic Ellipsometry. Thin Solid Films, 308-309, 268-272. http://dx.doi.org/10.1016/S0040-6090(97)00425-2

[11] Panwar, O.S., Khan, M.A., Bhattacharjee, B., Pal, A.K., Satyanarayana, B.S., Dixit, P.N., Bhattacharyya, R. and Khan, M.Y. (2006) Reflectance and Photoluminescence Spectra of as Grown and Hydrogen and Nitrogen Incorporated Tetrahedral Amorphous Carbon Films Deposited Using an S Bend Filtered Cathodic Vacuum Arc Process. Thin Solid Films, 515, 1597-1606. http://dx.doi.org/10.1016/j.tsf.2006.05.024

[12] Panwar, O.S., Khan, M.A., Kumar, M., Shivaprasad, S.M., Satyanarayana, B.S., Dixit, P.N., Bhattacharyya, R. and Khan, M.Y. (2008) Effect of High Substrate Bias and Hydrogen and Nitrogen Incorporation on Filtered Cathodic Vacuum Arc Deposited Tetrahedral Amorphous Carbon Films. Thin Solid Films, 516, 2331-2340. http://dx.doi.org/10.1016/j.tsf.2007.09.024

[13] Panwar, O.S., Khan, M.A., Kumar, S., Basu, A., Mehta, B.R. and Kumar, S. (2010) Effect of High Substrate Bias and Hydrogen and Nitrogen Incorporation of Spectroscopic Ellipsometric and Atomic Force Microscopic Studies of Tetrahedral Amorphous Carbon Films. Surface and Coatings Technology, 205, 2126-2133. http://dx.doi.org/10.1016/j.surfcoat.2010.08.119

[14] Weißmantel, S., Reiße, G. and Rost, D. (2004) Preparation of Super-Hard Amorphous Carbon Films with Low Internal Stress. Surface and Coatings Technology, 188-189, 268-273. http://dx.doi.org/10.1016/j.surfcoat.2004.08.070

[15] Materne, E.-M., Maschmeyer, I.J., Lorenz, A.K., Horland, R., Schimek, K.M.S., Busek, M., Sonntag, F., Lauster, R. and Marx, U. (2015) The Multi-Organ Chip-A Microfluidic Platform for Long-Term Multi-Tissue Coculture. Journal of Visualized Experiments, 98, e52526. http://dx.doi.org/10.3791/52526

[16] Maschmeyer, I., Lorenz, A.K., Schimek, K., Hasenberg, T., Ramme, A.P., Hübner, J., Lindner, M., Drewell, C., Bauer, S., Thomas, A., Sambo, N.S., Sonntag, F., Lauster, R. and Marx, U. (2015) A Four-Organ-Chip for Interconnected Long-Term Co-Culture of Human Intestine, Liver, Skin and Kidney Equivalents. In: Lab on a Chip.

[17] Schmieder, F., Schmieder, S., Eger, R., Friedrich, S., Werner, A., Danz, N., Marx, U. and Sonntag, F. (2012) Automated Universal Chip Platform for Fluorescence Based Cellular Assays. Biomedical Engineering/Biomedizinische Technik, 57, 340-343. http://dx.doi.org/10.1515/bmt-2012-4319

[18] Pedrotti, F., Pedrotti, L., Bausch, W. and Schmidt, H. (2008) Optik für Ingenieure. Springer, Berlin.

[19] Günther, K. (2014) Erzeugung und Anwendungspotential von mit dem PLD-Verfahren Hergestellten Superharten Amorphen Kohlenstoffschichten. PhD Dissertation, Shaker-Verlag, Maastricht. 